\title{
Quantum-Chemical Predictions of Absolute Standard Redox Potentials of Diverse Organic Molecules and Free Radicals in Acetonitrile
}

\author{
Yao Fu, Lei Liu,* Hai-Zhu Yu, Yi-Min Wang, Qing-Xiang Guo* \\ Department of Chemistry, University of Science and Technology of China, Hefei 230026, China
}

Email: leiliu@ustc.edu; qxguo@ustc.edu.cn

\section{Supporting Information}

Index

STable 1. Experimental and theoretical IP and $E^{0}$ values in $\mathrm{MeCN}$. $^{\text {a }}$

STable 2. Parameterization of the PCM model for $\mathrm{CH}_{3} \mathrm{CN}$.

Compete citations for Ref. 41 and 42. 
STable 1. Experimental and theoretical IP and $E^{0}$ values in $\mathrm{MeCN}{ }^{a}$

\begin{tabular}{|c|c|c|c|c|c|c|}
\hline Compound & $\begin{array}{c}\text { Exp. IP } \\
(\mathrm{eV})\end{array}$ & $\begin{array}{l}\text { Calc. IP } \\
(\mathrm{eV})\end{array}$ & $\begin{array}{c}\text { Calibrated IP } \\
(\mathrm{eV}) \\
(\text { cal. }+0.28 \mathrm{eV})\end{array}$ & $\begin{array}{c}E^{0} \text { vs. NHE } \\
(\mathrm{eV})\end{array}$ & $\begin{array}{l}\text { Calc. } E^{0} \\
\quad(\mathrm{eV})\end{array}$ & Ref. \\
\hline \multicolumn{7}{|c|}{ Alkanes } \\
\hline n-Pentane & $10.28 \pm 0.10$ & 9.87 & 10.15 & 3.39 & 3.57 & A1 \\
\hline 2-Methylpentane & 10.04 & 9.93 & 10.21 & 3.52 & 3.66 & A2 \\
\hline 3-Methylpentane & 10.04 & 9.82 & 10.1 & 3.43 & 3.42 & $\mathrm{~A} 40$ \\
\hline 2,2-Dimethylbutane & 10.07 & 9.89 & 10.17 & 3.79 & 3.80 & $\mathrm{~A} 2$ \\
\hline n-Hexane & $10.13 \pm 0.10$ & 9.64 & 9.92 & 3.35 & 3.54 & A1 \\
\hline n-Heptane & $9.93 \pm 0.10$ & 9.47 & 9.75 & 3.31 & 3.28 & A1 \\
\hline n-Octane & $9.80 \pm 0.15$ & 9.33 & 9.61 & 3.39 & 3.48 & A 40 \\
\hline & $8.70 \pm 0.01$ & 8.60 & 8.88 & 1.93 & 1.97 & A3 \\
\hline & - & 8.11 & 8.39 & 1.71 & 1.65 & A3 \\
\hline & - & 7.69 & 7.97 & 1.34 & 1.40 & A3 \\
\hline & - & 8.31 & 8.59 & 1.80 & 1.89 & A3 \\
\hline & - & 786 & 814 & 151 & 153 & A3 \\
\hline & & & & & & \\
\hline & - & 7.46 & 7.74 & 1.27 & 1.21 & A3 \\
\hline & - & 8.05 & 8.33 & 1.81 & 1.98 & A3 \\
\hline & - & 7.61 & 7.89 & 1.44 & 1.50 & A3 \\
\hline & - & 7.23 & 7.51 & 1.17 & 1.35 & A3 \\
\hline & - & 8.41 & 8.69 & 2.15 & 1.93 & A3 \\
\hline & $9.0 \pm 0.3$ & 8.48 & 8.76 & 2.36 & 2.22 & A3 \\
\hline & 8.72 & 8.17 & 8.45 & 1.74 & 1.91 & A3 \\
\hline & 8.18 & 8.00 & 8.28 & 1.78 & 1.73 & A3 \\
\hline & - & 8.08 & 8.36 & 1.86 & 1.91 & A3 \\
\hline & - & 7.98 & 8.26 & 1.78 & 1.7 & A3 \\
\hline & $8.1 \pm 0.3$ & 7.52 & 7.8 & 1.15 & 1.2 & A3 \\
\hline & $8.6 \pm 0.1$ & 8.40 & 8.68 & 1.97 & 2.1 & A3 \\
\hline$\triangle-$ & 8.47 & 8.24 & 8.52 & 1.98 & 1.91 & A3 \\
\hline Adamantane & $9.25 \pm 0.04$ & 8.86 & 9.14 & 2.93 & 2.95 & A8 \\
\hline
\end{tabular}




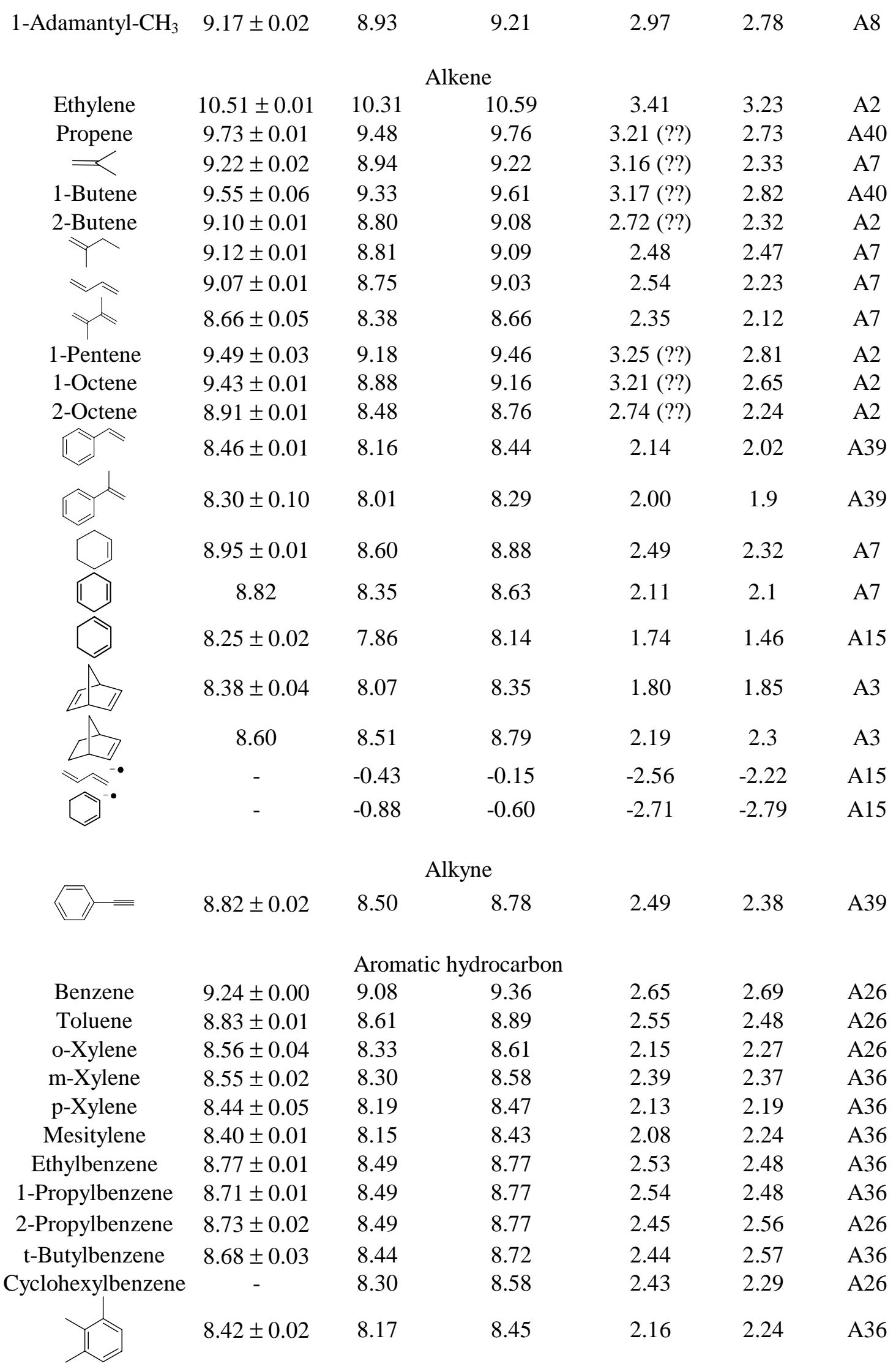




\begin{tabular}{|c|c|c|c|c|c|c|}
\hline & $8.27 \pm 0.01$ & 7.99 & 8.27 & 2.14 & 2.07 & A12 \\
\hline & $8.47 \pm 0.05$ & 7.83 & 8.11 & 2.07 & 2.02 & A26 \\
\hline & $8.06 \pm 0.03$ & 7.76 & 8.04 & 1.80 & 1.71 & A7 \\
\hline & 7.92 & 7.64 & 7.92 & 1.79 & 1.66 & A7 \\
\hline & $7.85 \pm 0.01$ & 7.49 & 7.77 & 1.71 & 1.62 & A7 \\
\hline & 8.35 & 8.02 & 8.30 & 2.08 & 2.09 & A12 \\
\hline & - & 7.73 & 8.01 & 1.83 & 1.93 & A4 \\
\hline (cis) & - & 7.81 & 8.09 & 1.91 & 1.76 & A12 \\
\hline (Trans) & - & 7.81 & 8.09 & 1.93 & 1.77 & A12 \\
\hline & - & 7.57 & 7.85 & 1.99 & 1.55 & A12 \\
\hline & - & 7.63 & 7.91 & 1.91 & 1.73 & $\mathrm{~A} 4$ \\
\hline & $8.6 \pm 0.1$ & 8.22 & 8.50 & 2.16 & 2.25 & A26 \\
\hline & $8.46 \pm 0.02$ & 8.20 & 8.48 & 2.14 & 2.1 & A26 \\
\hline & $8.14 \pm 0.01$ & 7.87 & 8.15 & 1.82 & 1.85 & A7 \\
\hline & $7.42 \pm 0.02$ & 7.23 & 7.51 & 1.18 & 1.33 & A7 \\
\hline & $8.16 \pm 0.13$ & 7.88 & 8.16 & 2.19 & 1.94 & A12 \\
\hline & $8.14 \pm 0.00$ & 7.87 & 8.15 & 1.85 & 1.85 & A7 \\
\hline & $7.96 \pm 0.03$ & 7.67 & 7.95 & 1.69 & 1.67 & A9 \\
\hline & $7.91 \pm 0.06$ & 7.69 & 7.97 & 1.67 & 1.69 & A9 \\
\hline & 8.11 & 7.54 & 7.82 & 1.59 & 1.62 & A9 \\
\hline & - & 7.50 & 7.78 & 1.60 & 1.55 & A9 \\
\hline & $8.12 \pm 0.10$ & 7.72 & 8.00 & 1.52 & 1.74 & A26 \\
\hline \multicolumn{7}{|c|}{ Amine } \\
\hline n-Propylamine & $8.5 \pm 0.1$ & 8.53 & 8.81 & 1.63 & 1.81 & A27 \\
\hline Diethylamine & $7.9 \pm 0.1$ & 7.89 & 8.17 & 1.52 & 1.5 & A20 \\
\hline n-Butylamine & 8.7 & 8.43 & 8.71 & 1.63 & 1.84 & A27 \\
\hline i-Butylamine & $8.5 \pm 0.1$ & 8.37 & 8.65 & 1.62 & 1.82 & A27 \\
\hline t-Butylamine & $8.5 \pm 0.1$ & 8.45 & 8.73 & 1.64 & 1.93 & A27 \\
\hline n-Pentylamine & 8.7 & 8.37 & 8.65 & 1.69 & 1.88 & A27 \\
\hline
\end{tabular}




\begin{tabular}{|c|c|c|c|c|c|c|}
\hline n-Nonylamine & - & 8.21 & 8.49 & 1.72 & 1.78 & A 27 \\
\hline Di-n-propylamine & $7.8 \pm 0.1$ & 7.75 & 8.03 & 1.43 & 1.54 & A 20 \\
\hline Di-i-propylamine & $7.6 \pm 0.1$ & 7.62 & 7.9 & 1.51 & 1.36 & A20 \\
\hline Di-n-butylamine & $7.69 \pm 0.03$ & 7.66 & 7.94 & 1.38 & 1.59 & A 20 \\
\hline Di-sec-butylamine & 7.6 & 7.53 & 7.81 & 1.40 & 1.64 & A 27 \\
\hline Trimethylamine & $7.85 \pm 0.05$ & 7.66 & 7.94 & 1.29 & 1.19 & A 27 \\
\hline Triethylamine & $7.53 \pm 0.10$ & 7.29 & 7.57 & 1.30 & 1.34 & A7 \\
\hline Tri-n-propylamine & 7.4 & 7.10 & 7.38 & 1.02 & 1.08 & A 27 \\
\hline & 8.86 & 8.34 & 8.62 & 1.59 & 1.64 & A27 \\
\hline & 8.41 & 8.10 & 8.38 & 1.09 & 1.59 & A 25 \\
\hline & $8.03 \pm 0.11$ & 7.79 & 8.07 & 1.36 & 1.33 & A 25 \\
\hline & 7.08 & 6.88 & 7.16 & 0.83 & 0.79 & A35 \\
\hline & $7.72 \pm 0.00$ & 7.54 & 7.82 & 1.21 & 1.23 & A7 \\
\hline & $7.80 \pm 0.10$ & 7.52 & 7.8 & 1.17 & 1.21 & A35 \\
\hline & 8.43 & 8.32 & 8.6 & 1.60 & 1.64 & A35 \\
\hline & $7.69 \pm 0.05$ & 7.44 & 7.72 & 1.24 & 1.23 & A 35 \\
\hline & $7.32 \pm 0.02$ & 7.25 & 7.53 & 1.14 & 1.06 & A 25 \\
\hline & $7.12 \pm 0.02$ & 7.05 & 7.33 & 1.10 & 1.13 & A31 \\
\hline & - & 7.78 & 8.06 & 1.52 & 1.53 & A29 \\
\hline & 7.56 & 7.17 & 7.45 & 1.16 & 1.06 & A22 \\
\hline & - & 6.96 & 7.24 & 0.91 & 0.94 & A11 \\
\hline & $6.1 \pm 0.1$ & 5.94 & 6.22 & 0.41 & 0.14 & A31 \\
\hline & - & 6.46 & 6.74 & 0.83 & 0.67 & A31 \\
\hline & 7.1 & 6.54 & 6.82 & 0.79 & 0.61 & A31 \\
\hline & - & 6.91 & 7.19 & 1.00 & 0.98 & A31 \\
\hline & $6.7 \pm 0.1$ & 6.51 & 6.79 & 0.84 & 0.58 & A31 \\
\hline & - & 6.73 & 7.01 & 0.99 & 0.85 & A 31 \\
\hline & 6.43 & 6.05 & 6.33 & 0.33 & 0.21 & A11 \\
\hline & 7.09 & 7.00 & 7.28 & 0.85 & 0.94 & A7 \\
\hline
\end{tabular}




\begin{tabular}{|c|c|c|c|c|c|c|}
\hline & $7.1 \pm 0.1$ & 7.06 & 7.34 & 0.95 & 1.03 & A7 \\
\hline & $7.19 \pm 0.05$ & 7.03 & 7.31 & 1.24 & 1.14 & A 25 \\
\hline & 7.32 & 6.96 & 7.24 & 0.81 & 0.82 & A20 \\
\hline & - & 7.23 & 7.51 & 0.80 & 1.2 & A20 \\
\hline & $7.53 \pm 0.03$ & 7.10 & 7.38 & 0.66 & 0.74 & A 21 \\
\hline & - & 6.77 & 7.05 & 0.62 & 0.58 & A 21 \\
\hline & - & 7.07 & 7.35 & 0.94 & 1.01 & A21 \\
\hline & - & 6.81 & 7.09 & 0.72 & 0.69 & A 21 \\
\hline & - & 6.20 & 6.48 & 0.47 & 0.2 & A 21 \\
\hline & - & 6.03 & 6.31 & 0.29 & 0.04 & A 21 \\
\hline & - & 6.61 & 6.89 & 0.94 & 0.53 & A 21 \\
\hline & - & 6.71 & 6.99 & 0.76 & 0.71 & A 21 \\
\hline $\mathrm{NMe}_{2}$ & - & 5.41 & 5.69 & -0.16 & 0.01 & A 21 \\
\hline$-\mathrm{CH}_{3}$ & - & 5.41 & 5.69 & -0.12 & -0.08 & A 21 \\
\hline & - & 6.77 & 7.05 & 0.82 & 0.78 & A 21 \\
\hline $\begin{array}{l}\mathrm{NMe}_{2} \\
\mathrm{CH}_{3}\end{array}$ & - & 11.18 & 11.46 & 1.54 & 1.71 & A 21 \\
\hline & & Alce & d ether & & & \\
\hline $\mathrm{CH}_{3} \mathrm{OH}$ & $10.84 \pm 0.01$ & 10.60 & 10.93 & 3.42 & 3.49 & A 34 \\
\hline & $10.48 \pm 0.07$ & 10.14 & 10.42 & 3.20 & 3.22 & A34 \\
\hline & $10.17 \pm 0.02$ & 9.77 & 10.05 & 3.19 & 3.13 & A34 \\
\hline & $9.99 \pm 0.05$ & 9.52 & 9.8 & 3.25 & 3.04 & A 34 \\
\hline & $9.88 \pm 0.03$ & 9.60 & 9.88 & 3.24 & 3.11 & A34 \\
\hline $\mathrm{HO}^{\sim}$ & 10.00 & 9.39 & 9.67 & 3.15 & 3.08 & A34 \\
\hline Phenol & $8.49 \pm 0.02$ & 8.32 & 8.6 & 1.61 & 2.02 & A7 \\
\hline 1-Adamantyl-OH & $9.09 \pm 0.05$ & 8.64 & 8.92 & 2.69 & 2.51 & A8 \\
\hline $\begin{array}{l}\text { 1-Adamantyl- } \\
\mathrm{CH}_{2} \mathrm{OH}\end{array}$ & 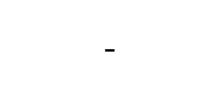 & 8.55 & 8.83 & 2.72 & 2.44 & A8 \\
\hline & 8.98 & 8.37 & 8.65 & 2.14 & 2.06 & A39 \\
\hline$\rangle$ & $8.20 \pm 0.05$ & 8.03 & 8.31 & 1.94 & 1.96 & A36 \\
\hline & 7.8 & 7.40 & 7.68 & 1.69 & 1.53 & $\mathrm{~A} 30$ \\
\hline
\end{tabular}




\begin{tabular}{|c|c|c|c|c|c|c|}
\hline $\mathrm{H}_{3} \mathrm{CO}-\mathrm{OCH}_{3}$ & $7.56 \pm 0.11$ & 7.27 & 7.55 & 1.51 & 1.42 & A11 \\
\hline & $8.2 \pm 0.1$ & 7.76 & 8.04 & 1.62 & 1.87 & A 30 \\
\hline & - & 7.13 & 7.41 & 1.66 & 1.34 & A 30 \\
\hline$-\mathrm{OCH}_{3}$ & $7.50 \pm 0.15$ & 7.00 & 7.28 & 1.36 & 1.24 & A30 \\
\hline & 7.96 & 7.40 & 7.68 & 1.73 & 1.6 & A30 \\
\hline & $9.19 \pm 0.01$ & 8.76 & 9.04 & 2.48 & 2.49 & A7 \\
\hline$\triangleleft$ & - & 7.32 & 7.6 & 1.59 & 1.42 & A4 \\
\hline & - & 7.30 & 7.58 & 1.70 & 1.29 & A4 \\
\hline 1-Adamantyl-OMe & - & 8.34 & 8.62 & 2.62 & 2.37 & A8 \\
\hline 0 & - & 7.67 & 7.95 & 1.70 & 1.68 & A11 \\
\hline & $8.10 \pm 0.10$ & 7.76 & 8.04 & 1.66 & 1.55 & A11 \\
\hline
\end{tabular}

Aldehyde, ketone, acid, and ester

$\begin{array}{lcccccc}\mathrm{O}_{\mathrm{H}}^{\bullet} & - & 0.51 & 0.79 & -1.25 & -1.23 & \mathrm{~A} 10 \\ & 0.39 \pm 0.05 & 0.56 & 0.84 & -1.44 & -1.44 & \mathrm{~A} 10\end{array}$

$\begin{array}{lllllll}\mathrm{I}_{\mathrm{CH}_{3}}^{\bullet} & 0.33 \pm 0.00 & 0.43 & 0.71 & -1.61 & -1.61 & \mathrm{~A} 10\end{array}$

$\begin{array}{lllllll}-7 \mathrm{COCH}_{3} & - & 8.14 & 8.42 & 2.19 & 2.13 & \mathrm{~A} 11\end{array}$

$\begin{array}{lllllll} & & & & & & \\ \mathrm{A} 11\end{array}$

(1)

$\begin{array}{lllllll}1 & -1.81 & 8.1 & 1.81 & \mathrm{~A} 11\end{array}$

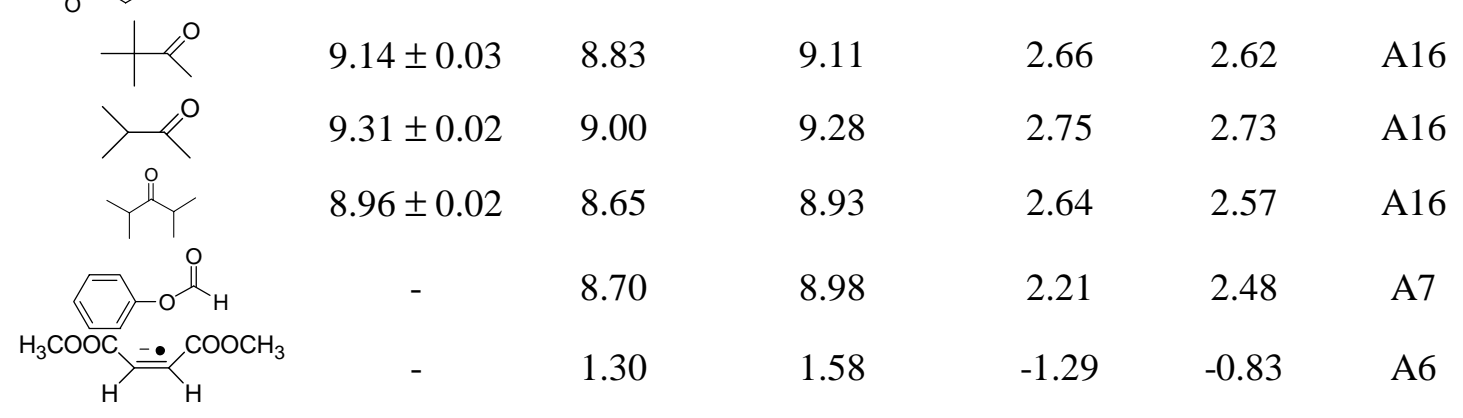



$\mathrm{H}_{\mathrm{H}}^{\mathrm{H}} \stackrel{\mathrm{COOCH}}{\mathrm{COOCH}_{3}}$
0.10
0.38
$-1.86$
$-1.89$
A6

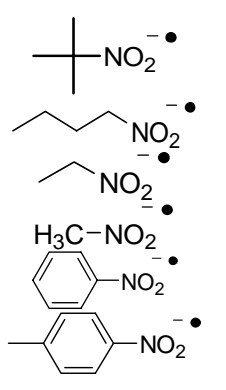

Nitro compound

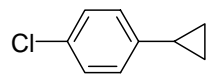

Chlorobenzene p-Chlorotoluene 1-Adamantyl-F 1-Adamantyl-Cl
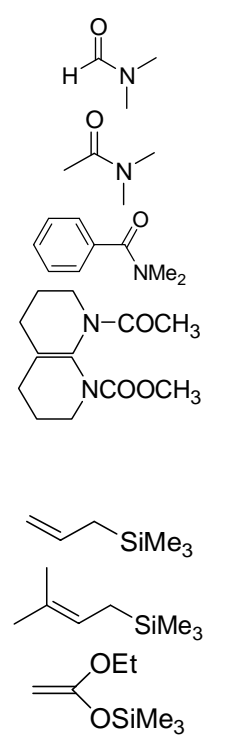

${ }_{-} \mathrm{OSiMe}_{3}$
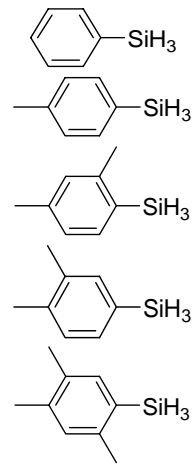

$\begin{array}{lllll}0.40 & 0.68 & -1.55 & -1.40 & \text { A5 } \\ 0.40 & 0.68 & -1.43 & -1.33 & \text { A5 } \\ 0.37 & 0.65 & -1.40 & -1.38 & \text { A5 } \\ 0.44 & 0.72 & -1.39 & -1.33 & \text { A5 } \\ 1.26 & 1.54 & -0.95 & -0.92 & \text { A5 } \\ 1.20 & 1.48 & -0.97 & -1.01 & \text { A5 }\end{array}$

-
-
-
-
-

Halogenated compound

\begin{tabular}{|c|c|c|c|c|c|}
\hline- & 7.97 & 8.25 & 2.21 & 2.02 & A4 \\
\hline $9.07 \pm 0.02$ & 8.85 & 9.13 & 2.64 & 2.58 & A 36 \\
\hline $8.69 \pm 0.03$ & 8.45 & 8.73 & 2.33 & 2.35 & A36 \\
\hline- & 9.13 & 9.40 & 3.21 & 2.92 & A8 \\
\hline 9.30 & 9.12 & 9.39 & 3.21 & 3.02 & A8 \\
\hline \multicolumn{6}{|c|}{ Amide } \\
\hline 9.13 & 9.06 & 9.34 & 2.53 & 2.51 & A 29 \\
\hline $9.20 \pm 0.05$ & 8.68 & 8.96 & 2.33 & 2.36 & A7 \\
\hline- & 8.34 & 8.62 & 2.28 & 2.35 & A 29 \\
\hline- & 8.34 & 8.62 & 2.12 & 2.25 & A 28 \\
\hline- & 8.28 & 8.65 & 2.20 & 2.26 & A28 \\
\hline
\end{tabular}

Silanes

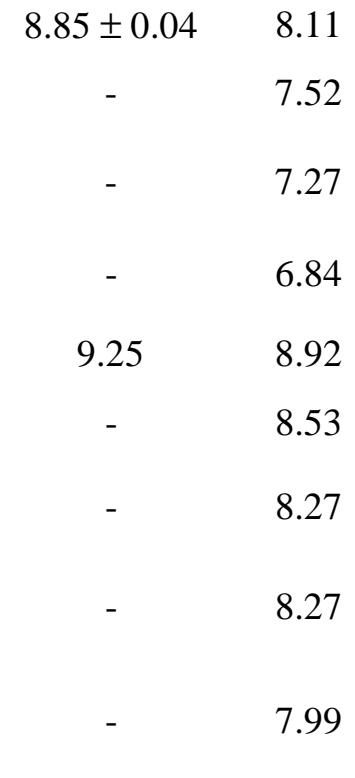

8.39

1.74

1.96

A24

1.63

1.51

A24

7.55

1.52

1.38

A24

7.12

1.14

0.93

A24

9.2

2.63

2.69

A37

8.81

2.51

2.39

A37

8.55

2.44

2.18

A37

8.55

2.41

2.18

A37

8.27

2.36

1.97

A37 


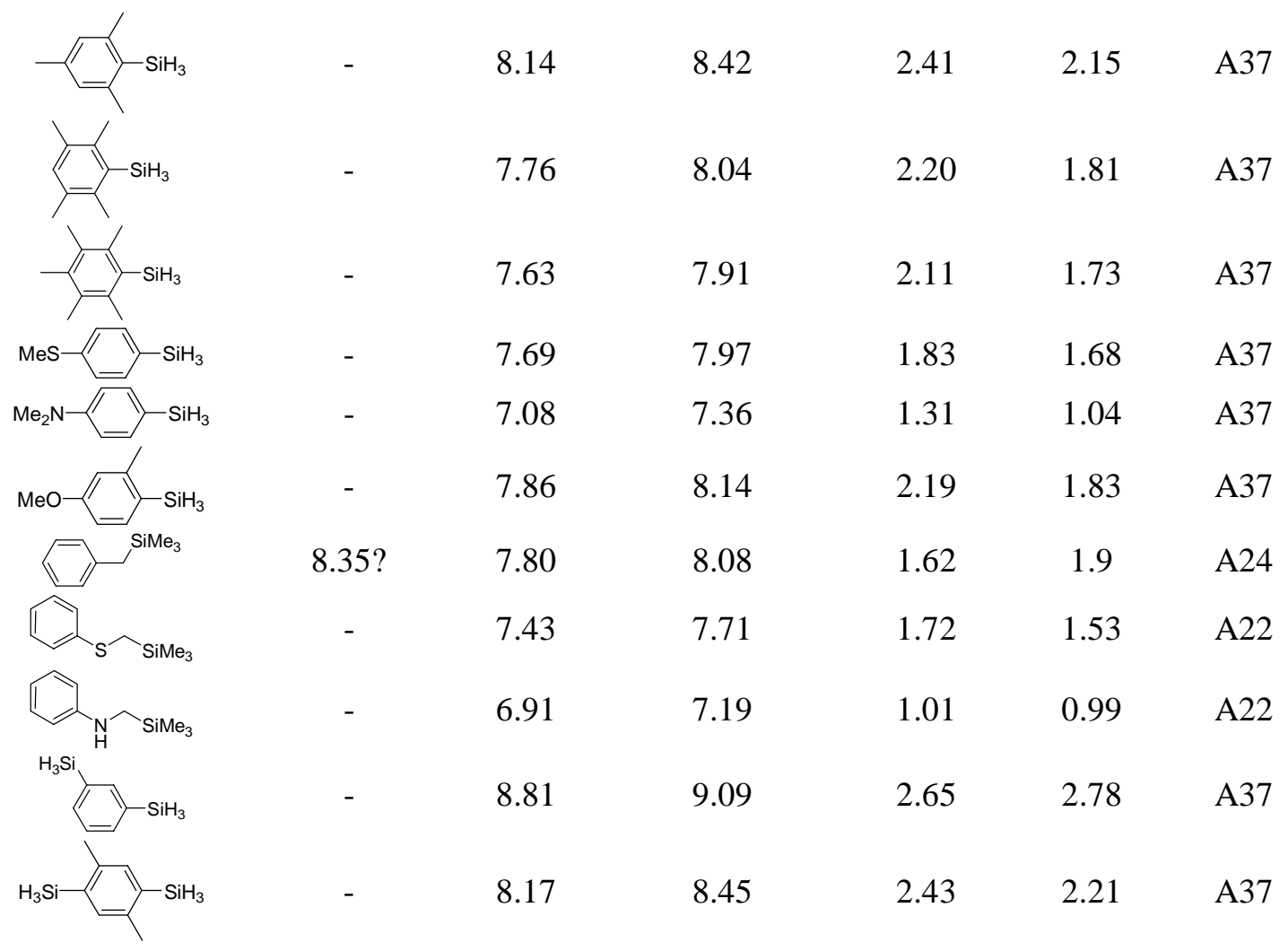

Thiol, sulfide, and sulfoxide

\begin{tabular}{|c|c|c|c|c|c|c|}
\hline $\mathrm{HS}$ & $9.20 \pm 0.01$ & 9.05 & 9.33 & 1.71(??) & 2.37 & A26 \\
\hline $\mathrm{SH}$ & $9.14 \pm 0.01$ & 8.91 & 9.19 & 1.91(??) & 2.48 & A7 \\
\hline $\mathrm{H}_{3} \mathrm{C}-\mathrm{S}-\mathrm{CH}_{3}$ & $8.69 \pm 0.02$ & 8.55 & 8.83 & 1.77 & 1.86 & A7 \\
\hline & $8.42 \pm 0.01$ & 8.25 & 8.53 & 1.92 & 1.96 & A7 \\
\hline & - & 7.22 & 7.50 & 1.2 & 1.24 & A3 \\
\hline & - & 6.63 & 6.91 & 0.81 & 0.89 & A3 \\
\hline & 9.10 & 8.70 & 8.98 & 2.24 & 1.98 & A7 \\
\hline & - & 8.67 & 8.95 & 2.31 & 2.25 & A28 \\
\hline
\end{tabular}

\begin{tabular}{|c|c|c|c|c|c|c|}
\hline \multicolumn{7}{|c|}{ Heterocycles } \\
\hline Pyrrole & $8.21 \pm 0.01$ & 8.10 & 8.38 & 1.33 & 1.52 & A26 \\
\hline Pyridine & $9.26 \pm 0.01$ & 9.02 & 9.3 & 2.39 & 2.73 & A26 \\
\hline Thiophene & $8.86 \pm 0.02$ & 8.71 & 8.99 & 2.17 & 2.22 & A 26 \\
\hline & - & 7.46 & 7.74 & 1.36 & 1.31 & A38 \\
\hline${ }^{\mathrm{N}-\mathrm{O}^{\ominus}}$ & - & 9.02 & 9.3 & 2.37 & 2.56 & A17 \\
\hline & $8.95 \pm 0.02$ & 8.80 & 9.08 & 2.51 & 2.45 & A17 \\
\hline
\end{tabular}




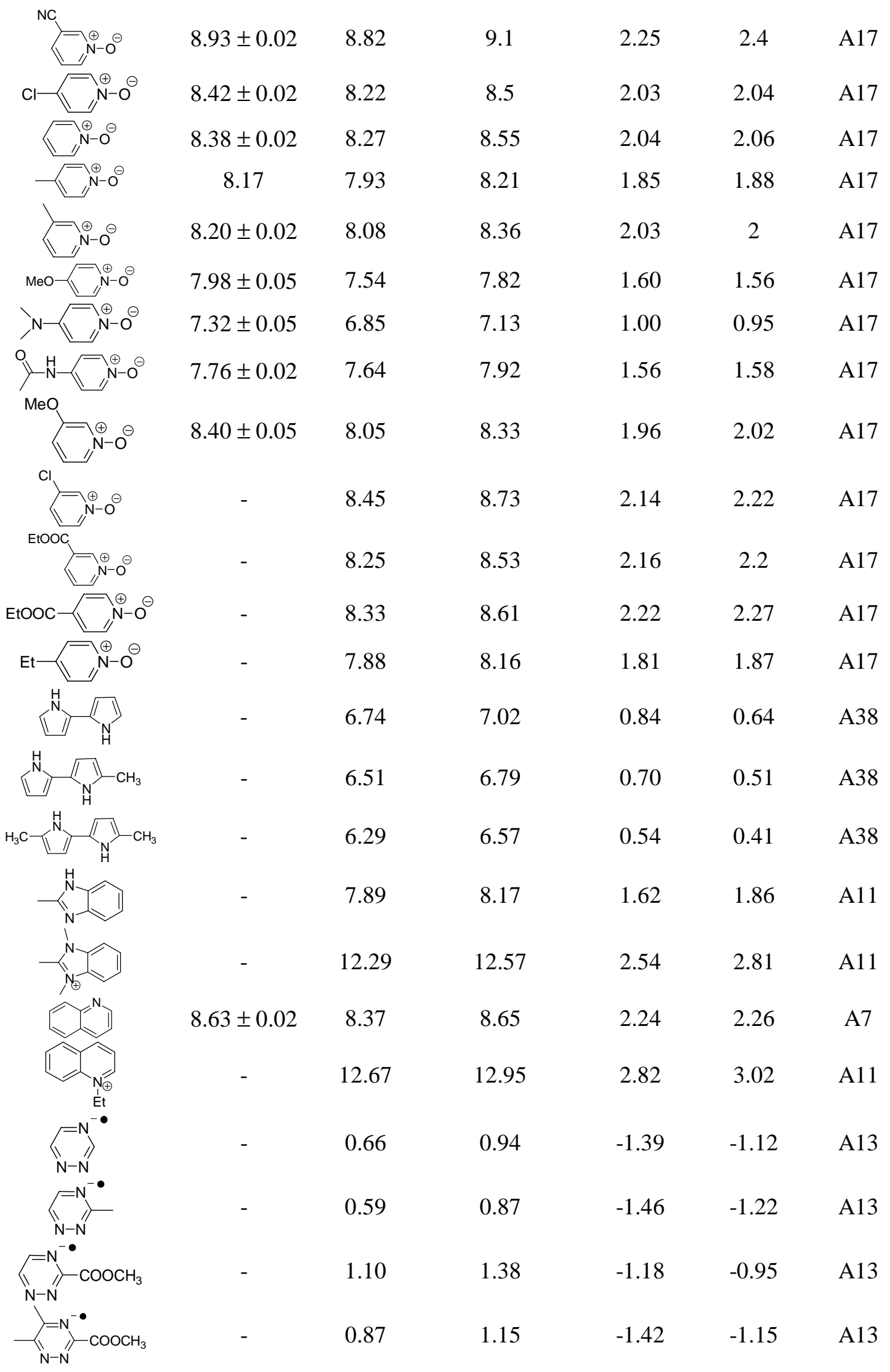




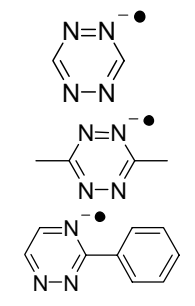

$\begin{array}{lllll}1.46 & 1.74 & -0.63 & -0.33 & \text { A13 } \\ 1.26 & 1.54 & -0.79 & -0.51 & \text { A13 } \\ 1.16 & 1.44 & -0.65 & -0.28 & \text { A13 }\end{array}$

\begin{tabular}{|c|c|c|c|c|c|c|}
\hline \multicolumn{7}{|c|}{ Hydrazine } \\
\hline & $7.29 \pm 0.05$ & 7.01 & 7.29 & 0.43 & 0.48 & A33 \\
\hline & $6.87 \pm 0.05$ & 6.75 & 7.03 & 0.52 & 0.59 & A18 \\
\hline & - & 6.64 & 6.92 & 0.51 & 0.43 & A18 \\
\hline & $6.63 \pm 0.05$ & 6.61 & 6.89 & 0.51 & 0.46 & A18 \\
\hline & $6.63 \pm 0.05$ & 6.59 & 6.87 & 0.51 & 0.47 & A18 \\
\hline & - & 6.47 & 6.75 & 0.50 & 0.13 & A18 \\
\hline & - & 6.37 & 6.65 & 0.49 & 0.29 & A18 \\
\hline & $6.75 \pm 0.05$ & 6.46 & 6.74 & 0.50 & 0.3 & A18 \\
\hline & $7.07 \pm 0.05$ & 6.70 & 6.98 & 0.68 & 0.53 & A18 \\
\hline & - & 6.59 & 6.87 & 0.76 & 0.55 & A18 \\
\hline & $6.59 \pm 0.05$ & 6.43 & 6.71 & 0.36 & 0.3 & A18 \\
\hline & $6.83 \pm 0.05$ & 6.64 & 6.92 & 0.55 & 0.27 & A18 \\
\hline & $6.37 \pm 0.05$ & 6.14 & 6.42 & 0.21 & 0.45 & A18 \\
\hline & $6.53 \pm 0.05$ & 6.25 & 6.53 & 0.39 & 0.31 & A18 \\
\hline & $6.51 \pm 0.05$ & 6.17 & 6.45 & 0.27 & 0.34 & A18 \\
\hline & $6.66 \pm 0.05$ & 6.41 & 6.69 & 0.30 & 0.22 & A18 \\
\hline & $6.45 \pm 0.05$ & 5.81 & 6.09 & 0.34 & 0.05 & A18 \\
\hline & - & 6.23 & 6.51 & 0.39 & 0.22 & A18 \\
\hline & $6.75 \pm 0.05$ & 6.44 & 6.72 & 0.42 & 0.54 & A18 \\
\hline & - & 6.51 & 6.79 & 0.52 & 0.39 & A32 \\
\hline & $6.71 \pm 0.05$ & 6.41 & 6.69 & 0.47 & 0.71 & A18 \\
\hline & - & 6.45 & 6.73 & 0.54 & 0.38 & A32 \\
\hline & - & 6.94 & 7.22 & 0.8 & 1.03 & A32 \\
\hline
\end{tabular}


$\begin{array}{lllllll}\substack{N^{N} \\ N^{N} \sim N^{-} \\-N^{-}} & 6.84 \pm 0.05 & 6.34 & 6.62 & 0.56 & 0.38 & \text { A32 }\end{array}$

\begin{tabular}{|c|c|c|c|c|c|c|}
\hline \multicolumn{7}{|c|}{ Azoalkanes } \\
\hline & $8.45 \pm 0.04$ & 8.35 & 8.63 & 2.21 & 2.09 & A23 \\
\hline & - & 8.12 & 8.40 & 2.09 & 2.05 & A23 \\
\hline & - & 7.87 & 8.15 & 1.83 & 1.77 & A23 \\
\hline & 7.95 & 7.75 & 8.03 & 1.69 & 1.62 & A23 \\
\hline & 7.73 & 7.48 & 7.76 & 1.58 & 1.52 & A23 \\
\hline & - & 8.09 & 8.37 & 2.29 & 2.19 & A23 \\
\hline & - & 8.43 & 8.71 & 2.54 & 2.25 & A23 \\
\hline \multicolumn{7}{|c|}{ Tetrazene } \\
\hline & 7.0 & 6.56 & 6.84 & 0.65 & 0.52 & A19 \\
\hline $\mathrm{N}-\mathrm{N}=\mathrm{\Lambda}$ & - & 6.23 & 6.52 & 0.56 & 0.54 & A19 \\
\hline
\end{tabular}

a. Experimental gas-phase ionization potentials are taken from NIST Standard Reference Database 69, March 1998 Release: NIST Chemistry WebBook (data compiled by J. E. Bartmess).

References:

A1: Clark, D. B.; Fleischmann, M.; Pletcher, D. J. Chem. Soc. Perkin Trans. 2 1973, 1578.

A2: Fleischmann, M.; Pletcher, D. Tetrahedron Lett. 1968, 60, 6255.

A3: Gassman, P. G.; Yamaguchi, R. Tetrahedron 1982, 38, 1113.

A4: Shono, T.; Matsumura, Y. J. Org. Chem. 1970, 35, 4157.

A5: Hojo, M.; Nishikawa, K.; Akita, Y.; Imai, Y. Bull. Chem. Soc. Jpn. 1986, 59, 3815.

A6: Tyssee, D. A.; Baizer, M. M. J. Org. Chem. 1974, 39, 2823.

A7: Miller, L. L.; Nordblom, G. D.; Mayeda, E. A. J. Org. Chem. 1972, 37, 916.

A8: Koch, V. R.; Miller, L. L. J. Am. Chem. Soc. 1973, 95, 8631.

A9: Pysk, E. S.; Yang, N. C. J. Am. Chem. Soc. 1963, 85, 2124.

A10: Loutfy, R. O.; Loutfy, R. O. J. Phys. Chem. 1973, 77, 336.

A11: Rudenko, A. P.; Zarubin, M. Y.; Pragst, F. J. Electroanal. Chem. Interfacial Electrochem. 1983, 151, 89.

A12: Guirado, G.; Fleming, C. N.; Lingenfelter, T. G.; Williams, M. L.; Zuihof, H.; Dinnocenzo, J. P. J. Am. Chem. Soc. 2004, 126, 14086.

A13: Troll, T. Electrochim. Acta 1982, 27, 1311.

A14: Bonesi, S. M.; Erra-Balsells, R. Perkin 2 2000, 1583.

A15: Breslow, R.; Johnson, R. W.; Krebs, A. Tetrahedron Lett. 1975, 3443. 
A16: Becker, J. Y.; Miller, L. L.; Siegel, T. M. J. Am. Chem. Soc. 1975, 97, 849.

A17: Loutfy, R. O. J. Chem. Phys. 1977, 66, 4781.

A18: Nelsen, S. F.; Peacock, V.; Weisman, G. R. J. Am. Chem. Soc. 1976, 98, 5269.

A19: Nelsen, S. F.; Peacock, V. E.; Kessel, C. R. J. Am. Chem. Soc. 1978, 100, 7017.

A20: Chow, Y. L.; Danen, W. C.; Nelsen, S. F.; Rosenblatt, D. H. Chem. Rev. 1978, 78, 243.

A21: Fritsch, J. M.; Weingarten, H.; Wilson, J. D. J. Am. Chem. Soc. 1970, 92, 4038.

A22: Yoshida, J.; Maekawa, T.; Murata, T.; Matsunaga, S.; Isoe, S. J. Am. Chem. Soc. 1990, 112, 1962.

A23: Nau, W. M.; Adam, W.; Klapstein, D.; Sahin, C.; Walter, H. J. Org. Chem. 1997, $62,5128$.

A24: Fukuzumi, S.; Fujita, M.; Otera, J.; Fujita, Y. J. Am. Chem. Soc. 1992, 114, 10271.

A25: Jonsson, M.; Wayner, D. D. M.; Lusztyk, J. J. Phys. Chem. 1996, 100, 17539.

A26: Loveland, J. W.; Dimeler, G. R. Anal. Chem. 1961, 33, 1196.

A27: Mann, C. K. Anal. Chem. 1964, 36, 2424.

A28: Shono, T. Tetrahedron 1984, 40, 811.

A29: Hall, L. R.; Iwamoto, R. T.; Hanzlik, R. P. J. Org. Chem. 1989, 54, 2446.

A30: Zweig, A.; Hodgson, W. G.; Jura, W. H. J. Am. Chem. Soc. 1964, 86, 4124.

A31: Zweig, A.; Lancaster, J. E.; Neglia, M. T.; Jura, W. H. J. Am. Chem. Soc. 1964, 86, 4130.

A32: Nelsen, S. F.; Hintz, P. J. J. Am. Chem. Soc. 1972, 94, 7108.

A33: Cauquis, G.; Chabaud, B.; Genies, M. J. Electroanal. Chem. 1972, 40, 6.

A34: Sundholm, G. Acta Chem. Scand. 1971, 25, 3188.

A35: Wawzonek, S.; McIntyre, T. W. J. Electrochem. Soc. 1967, 114, 1025.

A36: Neikam, W. C.; Dimeler, G. R.; Desmond, M. M. J. Electrochem. Soc. 1964, 111, 1190.

A37: Dhiman, A.; Becker, J. Y.; Minge, O.; Schmidbaur, H.; Muller, T. Organometallics 2004, 23, 1636.

A38: Andrieux, C. P.; Hapiot, P.; Audebert, P.; Guyard, L.; An, M. N. D.; Groenendaal, L.; Meijer, E. W. Chem. Mater. 1997, 9, 723.

A39: Katz, M.; Riemenschneider, P.; Wendt, H. Electrochim. Acta 1972, 17, 1595.

A40: Clark, D. B.; Fleischmann, M.; Pletcher, D. J. Electroanal. Chem. Interfacial Electrochem. 1973, 42, 133.

A41: Casado, J.; Gallardo, I.; Moreno, M. J. Electroanal. Chem. 1987, 219, 197. 
STable 2. Parameterization of the PCM model for $\mathrm{CH}_{3} \mathrm{CN}$.

Gas phase: $\quad$ B3LYP/6-311++G(2df,2p)//B3LYP/6-31+g(d)

Solvation model: $\quad$ B3LYP(PCM $/ 6-31+\mathrm{g} * *$ (radii=bondi)

\begin{tabular}{|c|c|c|c|c|c|c|c|}
\hline \multirow[t]{2}{*}{ Compound } & \multicolumn{7}{|c|}{$\Delta G_{\text {exchange }} / 2.303 \mathrm{RT}$} \\
\hline & Exp. p $K_{a}$ & $\mathrm{f}=1.10$ & $f=1.15$ & $\mathrm{f}=1.20$ & $\mathrm{f}=1.25$ & $\mathrm{f}=1.30$ & $f=1.35$ \\
\hline 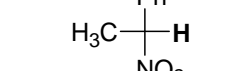 & 25.4 & -17.5 & -17.4 & -17.4 & -17.5 & -17.5 & -17.5 \\
\hline & 20.3 & -24.0 & -22.9 & -23.4 & -25.0 & -25.5 & -26.0 \\
\hline $\mathrm{H}_{3} \mathrm{COC}-\mathbf{H}$ & 24.4 & -19.3 & -19.4 & -18.5 & -18.2 & -18.5 & -18.3 \\
\hline & 27.2 & -14.5 & -14.5 & -14.1 & -14.1 & -13.8 & -13.9 \\
\hline & 25.0 & -16.2 & -16.4 & -16.3 & -16.1 & -16.2 & -16.4 \\
\hline & 23.8 & -17.0 & -17.2 & -17.5 & -17.7 & -18.0 & -18.6 \\
\hline & 22.7 & -19.0 & -19.1 & -19.3 & -19.4 & -19.7 & -20.2 \\
\hline & 20.9 & -22.3 & -21.6 & -22.0 & -22.3 & -22.8 & -23.3 \\
\hline & 22.3 & -21.3 & -21.0 & -20.6 & -20.3 & -20.1 & -19.9 \\
\hline & 20.7 & -21.8 & -21.6 & -21.5 & -21.7 & -21.3 & -21.3 \\
\hline & 18.8 & -25.7 & -25.7 & -25.0 & -25.4 & -25.4 & -25.2 \\
\hline & 15.8 & -28.2 & -28.2 & -27.3 & -28.4 & -28.6 & -28.6 \\
\hline & 22.7 & -20.9 & -20.8 & -20.3 & -20.0 & -19.7 & -20.0 \\
\hline & 24.6 & -16.2 & -16.6 & -16.5 & -16.6 & -16.4 & -16.3 \\
\hline $\mathrm{O}_{2} \mathrm{~N}-$ & 22.9 & -16.6 & -17.3 & -18.0 & -18.2 & -18.5 & -18.8 \\
\hline Slope & & 1.00 & 1.00 & 1.00 & 1.00 & 1.00 & 1.00 \\
\hline Intercept & & 42.5 & 42.5 & 42.3 & 42.6 & 42.6 & 42.8 \\
\hline$r$ & & 0.947 & 0.960 & 0.976 & 0.974 & 0.970 & 0.965 \\
\hline$s d$ & & 1.5 & 1.2 & 1.0 & 1.3 & 1.4 & 1.5 \\
\hline
\end{tabular}

Ref.

1. Stanczyk-Dunaj, M.; Galezowski, W.; Jarczewski, A. Can. J. Chem. 2002, 80, 1259.

2. Chantooni, M. K., Jr.; Kolthoff, I. M. J. Phys. Chem. 1976, 80, 1306.

3. Leito, I.; Kaljurand, I.; Koppel, I. A.; Yagupolskii, L. M.; Vlasov, V. M. J. Org. 
Chem. 1998, 63, 7863.

4. Kolthoff, I. M.; Chantooni, M. K., Jr.; Bhowmik, S. J. Am. Chem. Soc. 1968, 90, 23.

5. Chantooni, M. C., Jr.; Kolthoff, I. M. J. Phys. Chem. 1975, 79, 1176.

6. Ludwig, M.; Pytela, O.; Vecera, M. Collect. Czech. Chem. Commun. 1984, 49, 2593. 
Complete citations for Ref. 41 and 42.

41. Gaussian 98, Revision A.7, M.J. Frisch, G.W. Trucks, H.B. Schlegel, G.E. Scuseria, A. Robb, J.R. Cheeseman, V.G. Zakrzewski, J.A. Montgomery, Jr., R.E. Stratmann, J.C. Burant, S. Dapprich, J.M. Millam, A.D. Daniels, K.N. Kudin, M.C. Strain, O. Farkas, J. Tomasi, V. Barone, M. Cossi, R. Cammi, B. Mennucci, C. Pomelli, C. Adamo, S. Clifford, J. Ochterski, G.A. Petersson, P.Y. Ayala, Q. Cui, K. Morokuma, D.K. Malick, A.D. Rabuck, K. Raghavachari, J.B. Foresman, J. Cioslowski, J.V. Ortiz, A.G. Baboul, B.B. Stefanov, G. Liu, A. Liashenko, P. Piskorz, I. Komaromi, R. Gomperts, R.L. Martin, D.J. Fox, T. Keith, M.A. Al-Laham, C.Y. Peng, A. Nanayakkara, C. Gonzalez, M. Challacombe, P.M.W. Gill, B. Johnson, W. Chen, M.W. Wong, J.L. Andres, C. Gonzalez, M. Head-Gordon, E.S. Replogle, and J.A. Pople, Gaussian, Inc., Pittsburgh, PA, 1998.

42. Gaussian 03, Revision A.1, M. J. Frisch, G. W. Trucks, H. B. Schlegel, G. E. Scuseria, M. A. Robb, J. R. Cheeseman, J. A. Montgomery, Jr., T. Vreven, K. N. Kudin, J. C. Burant, J. M. Millam, S. S. Iyengar, J. Tomasi, V. Barone, B. Mennucci, M. Cossi, G. Scalmani, N. Rega, G. A. Petersson, H. Nakatsuji, M. Hada, M. Ehara, K. Toyota, R. Fukuda, J. Hasegawa, M. Ishida, T. Nakajima, Y. Honda, O. Kitao, H. Nakai, M. Klene, X. Li, J. E. Knox, H. P. Hratchian, J. B. Cross, C. Adamo, J. Jaramillo, R. Gomperts, R. E. Stratmann, O. Yazyev, A. J. Austin, R. Cammi, C. Pomelli, J. W. Ochterski, P. Y. Ayala, K. Morokuma, G. A. Voth, P. Salvador, J. J. Dannenberg, V. G. Zakrzewski, S. Dapprich, A. D. Daniels, M. C. Strain, O. Farkas, D. K. Malick, A. D. Rabuck, K. Raghavachari, J. B. Foresman, J. V. Ortiz, Q. Cui, A. G. Baboul, S. Clifford, J. Cioslowski, B. B. Stefanov, G. Liu, A. Liashenko, P. Piskorz, I. Komaromi, R. L. Martin, D. J. Fox, T. Keith, M. A. Al-Laham, C. Y. Peng, A. Nanayakkara, M. Challacombe, P. M. W. Gill, B. Johnson, W. Chen, M. W. Wong, C. Gonzalez, and J. A. Pople, Gaussian, Inc., Pittsburgh PA, 2003. 